\title{
Impedance matrix compression using an effective quadrature filter
}

\author{
J.-M.Huang, J.-L.Leou, S.-K.Jeng and J.-H.Tarng
}

\begin{abstract}
An effective quadrature mirror filter (QMF) proposed by Vaidyanathan has been used to solve 2D scattering problems. QMF has been popular for some time in digital signal processing, under the names of multirate sampling, wavelets, etc. In this work, the impulse response coefficients of QMF were used to construct the wavelet transform matrix. Using the matrix to transform the impedance matrices of $2 \mathrm{D}$ scatterers produces highly sparse moment matrices that can be solved efficiently. Such a presentation provides better sparsity than the celebrated and widely used Daubechies wavelets. These QMF coefficients are dependent on the filter parameters such as transition bandwidth and filter length. It was found that the sharper the transition bandwidth, the greater the reduction in nonzero elements of the impedance matrix. It also can be applied in the wavelet packet algorithm to further sparsify the impedance matrix. Numerical examples are given to demonstrate the effectiveness and validity of our finding.
\end{abstract}

\section{Introduction}

In computational electromagnetics, the method of moments (MoM) is a well established technique, but it produces a dense impedance matrix. Recently, the use of wavelets (or two-channel filter banks) for the solution of electromagnetic (EM) integral equations (IEs) has received attention [1-12]. The term 'wavelet' was introduced by Grossman and Morlet [13] to describe a square integrable function, appropriate translations and dilations of which form a basis for $\boldsymbol{L}^{2}(\mathbb{R})$. The term quadrature mirror filter (QMF) has been widely used in digital signal processing [14]. In this paper, we use these terms interchangeably. The most salient feature of wavelets is their ability to sparsify the impedance matrix. A classical basis function contains only magnitude/phase information, while the wavelet has not only magnitude/phase but also frequency (scale) information. Beylkin et al. [11, 12] first applied wavelets to the solution of IEs having smooth kernels (similar to those in electrostatic cases). For such problems, wavelets can be used to obtain a solution in $\mathrm{O}(N \log N)$ operations, where $N$ is the number of unknowns in the discretised IE. However, the kernel of IE in electrodynamics is an oscillatory type. Wavelets do not produce as dramatic a saving as smooth kernels. Most of the studies never pinpointed which kind of wavelet is more suitable to EM IEs with oscillatory kernels. Many papers use Daubechies wavelets to obtain matrix sparsification in MoM, but the discretion-

\section{(c) IEE, 2000}

IEE Proceedings online no. 20000361

DOI: 10.1049/ip-map:20000361

Paper first received 17th June 1999 and in revised form 4th February 2000

J.-M. Huang and J.-H. Tarng are with the Department of Communication Engineering, National Chiao-Tung University, Hsinchu, Taiwan, Republic of China

J.-L. Leou and S.-K. Jeng are with the Department of Electrical Engineering, National Taiwan University, Taipei, Taiwan, Republic of China ary choice of wavelets will not fully capitalise on the potential of the wavelet representation. The sparse moment matrix can be solved efficiently by sparse solvers and the computational cost is proportional to the nonzero elements. Hence, many researchers use different approaches to sparsify the matrix in MoM. Kim et al. [15] used the spectral domain wavelet transform (KDWT) to increase matrix sparsity. Golik [2] and Kim et al. [5] used wavelet packets (WPs) to sparsify the matrix further.

It is well known that taking the discrete wavelet transform of a signal is equivalent to filtering it by a constant-Q filter bank [14, 16]. In this work, 2D scattering problems are solved by fast wavelet transform in a filter-design perspective. The filter parameters such as transition bandwidth (TBW) and filter length determine the QMF coefficients. With these coefficients, wavelet transform matrix, $W$, can be constructed quickly to solve large matrix equations of MoM and leads to a better nonzero element reduction. This approach also highlights the fact that the discrete wavelet representation is determined solely by filter coefficients [17].

The filter-design approach essentially provides the same methodology as wavelets, but in a simpler, practical fashion. Many wavelet construction method constraints can be relaxed by applying this approach to sparsify the impedance matrix. For example, by relaxing the orthogonality condition, Cohen et al. [18] obtained a large family of biorthogonal exact reconstruction filters. Golik [19] and Xiang et al. [20] used the biorthogonal filter to obtain a higher compression rate in solving EM problems. By relaxing the minimum-phase condition of Daubechies wavelets, Huang et al. [21] used mix-phase wavelets to obtain better sparsification of the wavelet moment matrix. By relaxing the vanishing moment condition, Pan et al. [10] used Coifman wavelets on scattering problems. These Coifman wavelets and scaling function both have vanishing moments. However, in this study, we used Vaidyanathan filters [22], which do not satisfy any vanishing moment con- 
ditions [23]. Numerical wavelet transform (WT) and wavelet packet (WP) experiments are given to validate the relaxation of these conditions. Hence, in solving EM problems, the filter-design approach yields a greater degree of freedom for wavelet construction than the Daubechies wavelets approach.

\section{The effective construction of wavelet transform matrices and Vaidyanathan filters}

Many EM problems can be formulated as an inhomogeneous equation

$$
\mathcal{L} \mathrm{f}=\mathrm{g}
$$

where $\mathcal{L}$ is a linear operator, $\mathrm{g}$ is a known excitation and $\mathrm{f}$ is to be determined.

MoM can be used to solve eqn. 1. After expanding the unknown function in terms of $N$ known basis and weighting procedures, the algebraic linear equation systems can be obtained as

$$
Z I=V
$$

The discrete wavelet transform of a discrete signal is the signal multiplied by a unitary matrix. The unitary matrix arises from a wavelet basis for $L^{2}(\mathbb{R})$ in a manner which is addressed in the following portions of this Section. Let $W$ be a unitary wavelet matrix of order $N \times N$, the wavelet domain equation is formed as

$$
\tilde{Z} \tilde{I}=\tilde{V}
$$

where $\tilde{Z}=W Z W^{T}, \tilde{V}=W V$ and $\tilde{I}=\left(W^{T}\right)^{-1} I$.

Although the wavelet has no explicit analytical form, it can be obtained through the scaling function coefficients. The scaling function and wavelet function are defined as follows [14]:

$$
\begin{gathered}
\Phi(x)=\sqrt{2} \sum h_{n} \Phi(2 x-n) \\
\Psi(x)=\sqrt{2} \sum g_{n} \Psi(2 x-n) \\
g_{n}=(-1)^{1-n} h_{1-n} \quad n \in \mathbb{Z}
\end{gathered}
$$

where $h_{n}$ and $g_{n}$ are the coefficients of scaling and wavelet functions, respectively. $h_{n}$ and $g_{n}$ are, respectively, the Vaidyanathan [22] low- and highpass filter coefficients of QMF.

To compare different orthogonal wavelets, the $W$ matrix must be implemented quickly. For a vector $s^{0}$ of size $N=$ $2^{n}$, a $N \times N$ matrix $W_{n}$ is decomposed as

$$
W_{n}=\left[\begin{array}{l}
H_{n} \\
G_{n}
\end{array}\right]
$$

where $H_{n}$ and $G_{n}$ are matrices of order $2^{n-1} \times 2^{n}$ called the low- and highpass filters with impulse responses $h_{n}$ and $g_{n}$, respectively. For example, if $n=4$ then $H_{n}$ and $G_{n}$ matrices are

$$
\begin{array}{r}
H_{n}=\left[\begin{array}{cccccccc}
h_{1} & h_{2} & h_{3} & h_{4} & 0 & \cdots & & 0 \\
0 & 0 & h_{1} & h_{2} & h_{3} & h_{4} & \cdots & 0 \\
\vdots & \vdots & & \ddots & & & \vdots & \vdots \\
0 & \cdots & & & h_{1} & h_{2} & h_{3} & h_{4} \\
h_{3} & h_{4} & \cdots & & & & h_{1} & h_{2}
\end{array}\right] \\
G_{n}=\left[\begin{array}{cccccccc}
g_{1} & g_{2} & g_{3} & g_{4} & 0 & \ldots & & 0 \\
0 & 0 & g_{1} & g_{2} & g_{3} & g_{4} & \cdots & 0 \\
\vdots & \vdots & & \ddots & & & \vdots & \vdots \\
0 & \cdots & & & g_{1} & g_{2} & g_{3} & g_{4} \\
g_{3} & g_{4} & \cdots & & & & g_{1} & g_{2}
\end{array}\right]
\end{array}
$$

The filter coefficients are wrapped around to form a circulant matrix. $W_{n} s^{0}$ decomposes $s^{0}$ into a smooth (average) part $s^{1}$ and a detailed (difference) part $d^{\mathrm{I}}$.

Continuing the process of recursive decomposition of smooth vectors $g^{j}$, we obtain the discrete wavelet transform of the vector $s^{0}$. In the matrix notation, $W s^{0}=W_{n-\ell} \ldots$ $W_{n-1} W_{n} s^{0}=\left[s^{\ell}, d^{\ell}, \ldots, d^{l}\right]$ and $W_{n-j}$ is a block-diagonal matrix of the form

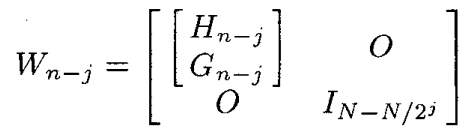

where $I_{N-N / 2}^{j}$ is an identity matrix of rank $N-N / 2^{j}$.

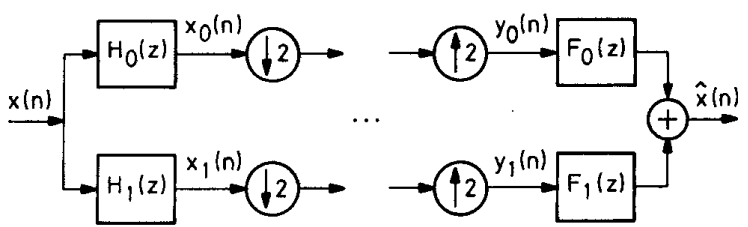

Fig. 1 A two-channel QMF bank

Fig. 1 illustrates a QMF bank that functions as a wavelet transform. The left-hand side of the figure corresponds to the wavelet transform and the right-hand side corresponds to the inverse wavelet transform. In the left part, the signal $x(n)$ is split and passed to two channels that have a lowpass filter, $H_{0}(z)$, of bandwidth $\pi / 2[0 \leq \omega \leq \pi / 2]$, and a highpass filter, $H_{1}(z)$, of bandwidth $\omega=\pi / 2$ to $\pi$, respectively. Both subband signals are undersampled (or decimated) by a factor of two. In the other part, inverse processes are shown. The choice

$$
\begin{gathered}
H_{1}(z)=z^{-(N-1)} H_{0}\left(-z^{-1}\right) \\
F_{0}(z)=z^{-(N-1)} H_{0}\left(z^{-1}\right) \\
F_{1}(z)=z^{-(N-1)} H_{1}\left(z^{-1}\right)
\end{gathered}
$$

results in a perfect reconstruction since aliasing is cancelled. For readers without a strong background in QMF, excellent discussions of the subject can be found in [14, 24].

Obviously, $H_{0}(z)$ and $H_{1}(z)$ are crucial for the design of the QMF bank. These transfer functions can be implemented by a new family of lattice structures [25] as shown in Fig. 2. Detailed construction methods of the impulse response coefficients of the above transfer functions are available [22]. These coefficients determine the associated $W$-matrix of WT for 2D scattering problems. We briefly recapitulate the design procedure of the impulse response coefficients.

(1) Design $H_{0}(z)$ based on lattice structure and its relation to the halfband filters.

(2) The desired frequency responses of the transfer functions $\hat{P}_{M-1}(z)$ and $\hat{Q}_{M-1}(z)$ are shown in Fig. 3. The object function is formulated as

$$
\prod_{o b j}=\int_{\omega_{s}}^{\pi}\left|\hat{P}_{M-1}\left(e^{j \omega}\right)\right|^{2} d \omega
$$

Optimise lattice $\alpha_{m}$ so as to minimise the stopband energy, $\Pi_{o b j}$ of the filter for various $\omega_{s}$ and order $M$.

(3) Compute the impulse response coefficients from the given set of lattice coefficients $\alpha_{m}$. A short program in [22] can be applied. 


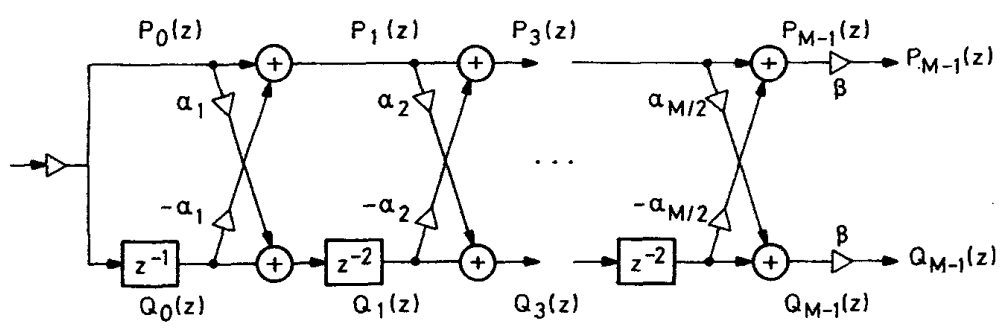

Fig.2 QMF lattice

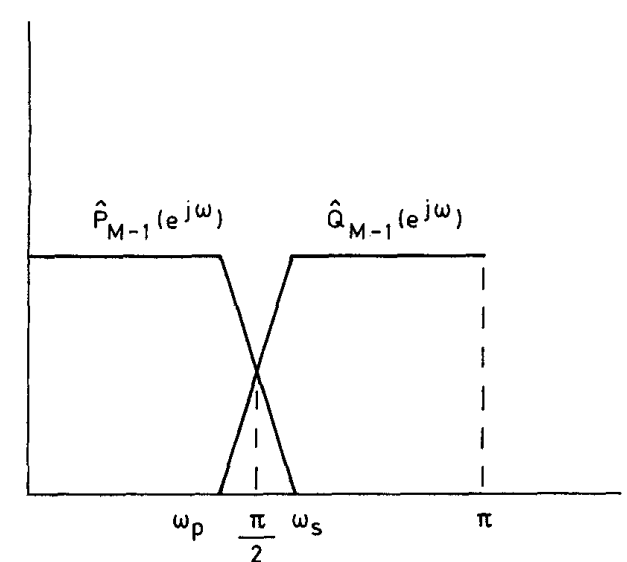

Fig.3 Desired frequency responses of the lattice transfer functions

With the filter impulse response coefficients, the $W$ matrix can be formed quickly. Some filter specifications are given in Table 1 (summarised from Table I-VII [22]). Their associated impulse response coefficients are listed in Table 2.

Tables 1: Specifications of lattice filters [22]

\begin{tabular}{lll}
\hline Filter number & $\omega_{s}$ specified, $\pi$ rad & $\begin{array}{l}\text { Transition bandwidth } \\
\left(\omega_{s}-0.5 \pi\right) /(2 \pi)\end{array}$ \\
\hline $16 \mathrm{a}$ & 0.78 & 0.1407 \\
$24 \mathrm{~b}$ & 0.70 & 0.1010 \\
$32 \mathrm{c}$ & 0.62 & 0.0617 \\
$48 \mathrm{f}$ & 0.60 & 0.0510 \\
\hline
\end{tabular}

\section{Wavelet packets}

The WT tree follows the rigid constant-Q structure that moves progressively towards the lowest spectral content. However, at each decomposition level two branches are possible. Either low-frequency or high-frequency or only high-frequency can be decomposed. There are many possible tree structures, and correspondingly many transformations, each producing a different set of basis functions. By adaptively selecting transformation bases based on a cost function at every node of the decomposition, the WP transform [23] can determine a basis which suits the problem. Note that the transformation matrix remains orthogonal.

Many different types of basis selection algorithm (top down or bottom up) and cost function (entropy, log-energy or $\ell^{\mathrm{p}}$ norm) can be chosen in WP. For verification of the QMF, we adopted the same scheme as [2]. Using the incident wave vector as the top node of the WP decomposition tree, if the cost of the parent node vector is smaller than the norm of its two children, this branch is not decomposed
Tables 2: Impulse response coefficients for various Vaidyanathan filters

\begin{tabular}{|c|c|c|c|}
\hline $16 \mathrm{~A}$ & $24 B$ & $32 \mathrm{C}$ & $48 F$ \\
\hline$-4.1065 e-04$ & $-6.2906 e-05$ & $-2.1472 e-03$ & $1.1199 \mathrm{e}-04$ \\
\hline $1.9297 \mathrm{e}-03$ & $3.4363 \mathrm{e}-04$ & $1.0623 \mathrm{e}-02$ & $-6.7839 e-04$ \\
\hline$-1.0154 e-03$ & $-4.5396 \mathrm{e}-04$ & $-1.9673 e-02$ & $1.6759 \mathrm{e}-03$ \\
\hline$-9.1157 e-03$ & $-9.4490 e-04$ & $8.7812 \mathrm{e}-03$ & $-1.7081 e-03$ \\
\hline $1.3238 \mathrm{e}-02$ & $2.8438 \mathrm{e}-03$ & $2.1905 e-02$ & $-6.3173 e-04$ \\
\hline $2.1511 \mathrm{e}-02$ & $7.0814 \mathrm{e}-04$ & $-2.5044 \mathrm{e}-02$ & $3.0020 \mathrm{e}-03$ \\
\hline$-5.1296 \mathrm{e}-02$ & $-8.8391 \mathrm{e}-03$ & $-2.2323 e-02$ & $-7.0100 \mathrm{e}-04$ \\
\hline$-3.2633 e-02$ & $3.1538 \mathrm{e}-03$ & $4.3529 \mathrm{e}-02$ & $-4.3027 e-03$ \\
\hline $1.2924 \mathrm{e}-01$ & $1.9687 \mathrm{e}-02$ & $2.2452 \mathrm{e}-02$ & $2.6101 e^{-03}$ \\
\hline $4.1629 \mathrm{e}-02$ & $-1.4853 \mathrm{e}-02$ & $-6.6621 \mathrm{e}-02$ & $5.8123 \mathrm{e}-03$ \\
\hline$-2.6276 \mathrm{e}-01$ & $-3.5470 \mathrm{e}-02$ & $-2.3242 e-02$ & $-5.4870 e^{-03}$ \\
\hline$-1.0579 \mathrm{e}-01$ & $3.8742 \mathrm{e}-02$ & $9.5931 \mathrm{e}-02$ & $-7.4700 \mathrm{e}-03$ \\
\hline $4.8664 \mathrm{e}-01$ & $5.5893 \mathrm{e}-02$ & $2.7635 \mathrm{e}-02$ & $9.6763 \mathrm{e}-03$ \\
\hline $7.0595 \mathrm{e}-01$ & $-7.7710 \mathrm{e}-02$ & $-1.3344 e-01$ & $9.1201 \mathrm{e}-03$ \\
\hline $3.9334 \mathrm{e}-01$ & $-8.3929 e-02$ & $-4.3460 \mathrm{e}-02$ & $-1.5469 \mathrm{e}-02$ \\
\hline \multirow[t]{27}{*}{$8.3705 e-02$} & $1.3197 \mathrm{e}-01$ & $1.8146 \mathrm{e}-01$ & $-1.0582 \mathrm{e}-02$ \\
\hline & $1.3508 \mathrm{e}-01$ & $9.1260 \mathrm{e}-02$ & $2.3099 \mathrm{e}-02$ \\
\hline & $-1.9445 e-01$ & $-2.3632 \mathrm{e}-01$ & $1.1712 \mathrm{e}-02$ \\
\hline & $-2.6349 \mathrm{e}-01$ & $-2.2922 \mathrm{e}-01$ & $-3.2754 \mathrm{e}-02$ \\
\hline & $2.0161 \mathrm{e}-01$ & $2.3238 \mathrm{e}-01$ & $-1.2486 \mathrm{e}-02$ \\
\hline & $6.3560 \mathrm{e}-01$ & $6.1712 \mathrm{e}-01$ & $4.4606 \mathrm{e}-02$ \\
\hline & $5.7280 \mathrm{e}-01$ & $5.5528 \mathrm{e}-01$ & $1.3109 \mathrm{e}-02$ \\
\hline & $2.5018 \mathrm{e}-01$ & $2.5553 \mathrm{e}-01$ & $-5.8857 e-02$ \\
\hline & $4.5799 \mathrm{e}-02$ & $5.1651 \mathrm{e}-02$ & $-1.4179 \mathrm{e}-02$ \\
\hline & & & $7.5814 \mathrm{e}-02$ \\
\hline & & & $1.6973 e-02$ \\
\hline & & & $-9.5924 e-02$ \\
\hline & & & $-2.3979 e-02$ \\
\hline & & & $1.1965 \mathrm{e}-01$ \\
\hline & & & $4.0003 e-02$ \\
\hline & & & $-1.4669 \mathrm{e}-01$ \\
\hline & & & $-7.4524 \mathrm{e}-02$ \\
\hline & & & $1.7258 \mathrm{e}-01$ \\
\hline & & & $1.4637 \mathrm{e}-01$ \\
\hline & & & $-1.7505 \mathrm{e}-01$ \\
\hline & & & $-2.8850 \mathrm{e}-01$ \\
\hline & & & $5.6071 \mathrm{e}-02$ \\
\hline & & & $4.9411 \mathrm{e}-01$ \\
\hline & & & $5.9620 \mathrm{e}-01$ \\
\hline & & & $3.8140 \mathrm{e}-01$ \\
\hline & & & $1.3772 \mathrm{e}-01$ \\
\hline & & & $2.2736 \mathrm{e}-02$ \\
\hline
\end{tabular}


further. Otherwise, continue to decompose the tree from this node. A commonly used cost function is

$$
\mathrm{C}(x)=\sum_{m}\left|x_{m}\right| \quad x=\left[x_{1}, x_{2}, \ldots, x_{M}\right]^{T}
$$

where $x$ is a vector of transformation coefficients related to the node.

Since this top-down algorithm may not expand the full tree, it does not guarantee finding the best tree for the given incident vector and cost. The selected basis is a near-best tree. If the best basis selection [26] is concerned, the search time will increase. The effect of basis selection algorithm and cost function is beyond the present discussion. Our focus is to evaluate the performance of different QMFs using the same WP algorithm.

Once the tree structure has been determined, the associated $W$ matrix is obtained and applied to the matrix $Z$. The remaining procedure is the same as the WT procedure.

\section{Numerical results}

In this Section we use the fast construction method in Section 2 and WP in Section 3 to solve EM scattering IE. A 2D perfect electric conductor (PEC) of an L-shaped scatterer and cylinder are chosen to validate the QMF using the same discretisation size (10 points per $\lambda)$ and relative residual error. The two structures are taken from [1]. A discretisation size of $0.1 \lambda$ is often sufficient to model the current on the scatterer. If finer discretisation were used, a greater sparsity would be obtained but at the cost of more unknowns for a given problem. Detailed explanations can be found in $[1,4]$. By changing the physical size of the scatterer proportionally, the MoM matrices with sizes in the range $N=256-2048$ are obtained. To sparsify the transformed impedance matrices, the elements of the matrices are held at

$$
\tau=\frac{\varepsilon}{N}\|Z\|_{\infty}=\frac{\varepsilon}{N} \cdot \max _{i} \sum_{j=1}^{N}\left|Z_{i j}\right|
$$

This level has been adopted by [12]. The advantage is that the relative error of the solution will appear under a predictable limit. The relative residual error is defined by

$$
\text { Residual error }=\frac{\left\|\tilde{V}-\tilde{Z} \tilde{J}_{c}\right\|}{\|\tilde{V}\|} \times 100 \%
$$

where $\tilde{J}_{c}$ determined from the threshold matrix $\tilde{Z}$. Here we fix the residual error at $(1 \pm 0.1) \%$.

In solving the problem of a TM $\left(E_{Z}\right)$ polarised wave scattered by a $2 \mathrm{D}$ conducting scatterer, the scattering characteristics are obtained from the surface current density $J_{Z}$ excited by an incident wave $E_{Z}^{i n c}$. The unknown current density $J_{Z}$ determined from an electric field integral equation as follows:

$$
\oint_{c} J_{Z}\left(r^{\prime}\right) G\left(r, r^{\prime}\right) d r^{\prime}=-E_{Z}^{i n c}(r)
$$

where $c$ represents the surface of the scatterer.

Dividing the PEC body surface into subdomains and employing Galerkin's technique, the IE is reduced to a matrix equation. The matrix equation is then solved using the WT method in Section 2. Different QMF coefficients listed in Table 2 are used to generate the associated $W$ matrices. Sampled QMF with different specifications are chosen to compare with the widely used Daubechies wavelet. The sparsified results for the cylindrical scatterer are presented in Fig. 4 for various filters matrices. The sparsity using these QMF is better than that of the Daubechies wavelet. Fig. 4 illustrates that the sharper the TBW of the filters, the greater the nonzero reduction element in the impedance matrix. The TBW of Daubechies wavelets is 4/ $\sqrt{ } N$ [14]. The TBW of Daubechies 16 is 1 and this is the largest of those filters. Therefore, the sparsity performance of Daubechies 16 is the worst. This suggests that TBW is an important factor if wavelets are chosen using the filterdesign concept.

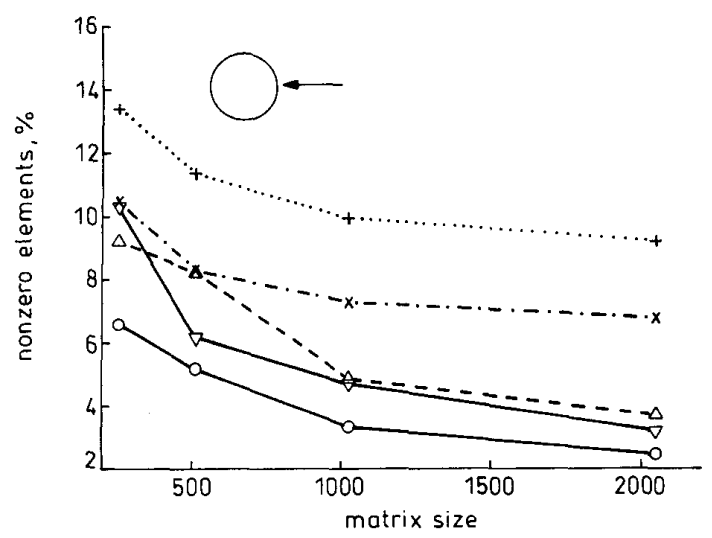

Fig. 4 Percentage of nonzero elements as a function of matrix size by different filters for a cylindrical scatterer

$+\ldots . .+$ Daubechies 16

$x-\cdots \times$ filter $16 \mathrm{a}$

$\triangle--\Delta$ filter 24

$\nabla-\nabla$ filter 32

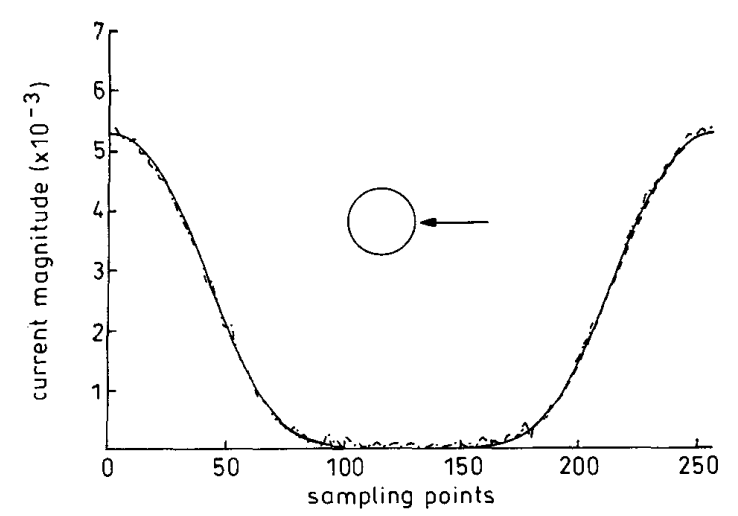

Fig.5 Induced surface current for a cylinder with $\mathrm{ka}=25.6, \mathrm{~N}=256$

MoM solution WT-MoM by $48 \mathrm{f}$ exact [27]

Fig. 5 displays the induced surface current of a cylinder, which was computed by the WT MoM, conventional MoM and exact solution. It was found that the solution accuracy of the MoM and the WT MoM is almost the same when the residual error is fixed at $1 \%$. The exact solution for a circular cylinder is given by [27]

$$
J_{Z}=\frac{-2 E_{0}}{\omega \mu \pi a} \sum_{n=-\infty}^{\infty} \frac{j^{-n} e^{j n \varphi}}{H_{n}^{(2)}(k a)}
$$

where $n=300$ in the numerical computation because the magnitude for $n>300$ is negligible.

A similar numerical experiment was carried out for an L-shaped scatterer, the sparsity is shown in Fig. 6. It is obvious that the filter length and TBW have important effects on the sparsity. Filter $32 \mathrm{c}$ has better performance at matrix size $N=256$ for its shorter length and sharper 
TBW. Filter $48 \mathrm{f}$ has better sparsity with the increase of matrix size $N$. This is because the sharp TBW of the filter and the relative importance of the geometrical corners diminish. This figure also suggests that TBW is a crucial point in wavelet selection for large problems. Fig. 7 is the induced surface current, and the difference between MoM and WT MoM is negligible.

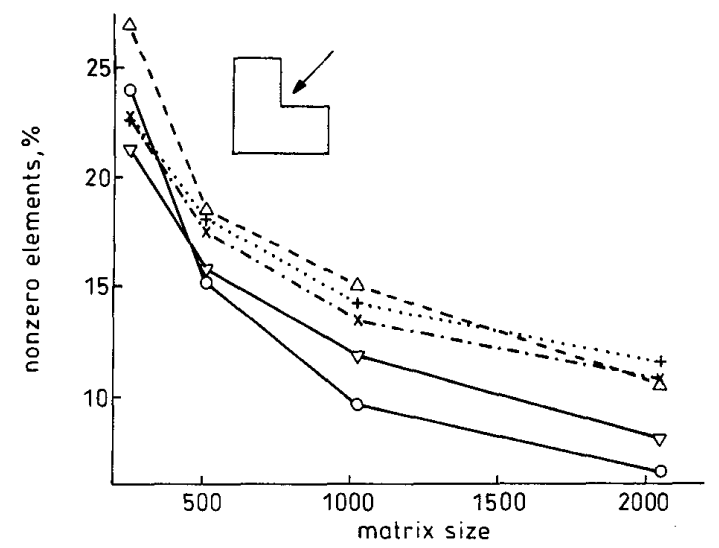

Fig. 6 The percent of nonzero elements as a function of matrix size by different filters for an L-shaped scatterer

$+\cdots+.+$ Daubechies 16

$\times-\cdots \cdot \times$ filter $16 \mathrm{a}$

$\Delta---\Delta$ filter $24 b$

$\nabla-\nabla$ filter $32 \mathrm{c}$

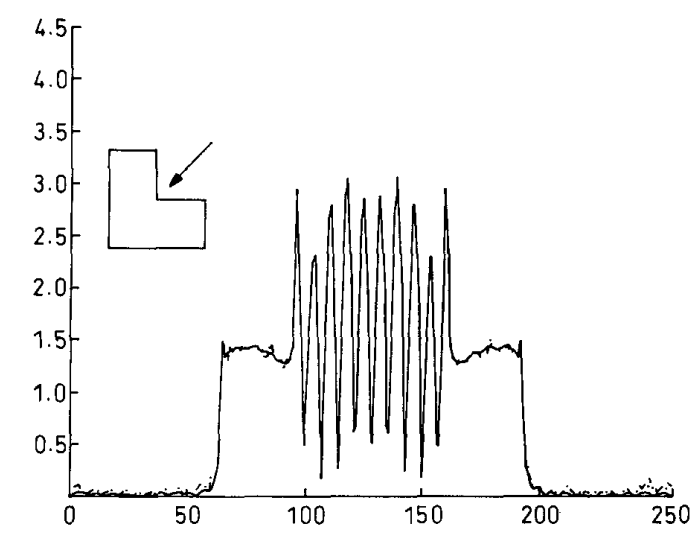

Fig.7 Induced surface current for an L-shaped scatterer with contour = $25.6 \lambda ; N=256$

-..-- MTM solution

The WP algorithm in Section 3 can be used to sparsify the impedance matrix further. The numerical results are shown in Figs. 8 and 9 for the cylinder and L-shaped scatterers, respectively. To demonstrate the improvement in sparsity, the curves using WT are added in these figures. It is obvious that Filter $48 \mathrm{f}$ has better performance because its TBW is the sharpest. In the cylindrical case, its WT performance is even better than the WP of the Daubechies 16 wavelet. This suggests that the discretionary choice of the wavelet must increase computing time to obtain the same sparsity using a more sophisticated algorithm like WP. In the L-shaped scatterer case, the sparsity is easily affected by the corners of scatterer at small matrix size $N$ and better at larger $N$. However, the direct solution of conventional MoM equations is prohibitively expensive for large-scale scattering problems. Therefore, the selection of wavelets with sharper TBW and shorter length is still valuable for sparsity improvement. This is because the effects of geometrical bending diminish for large-scale problems.

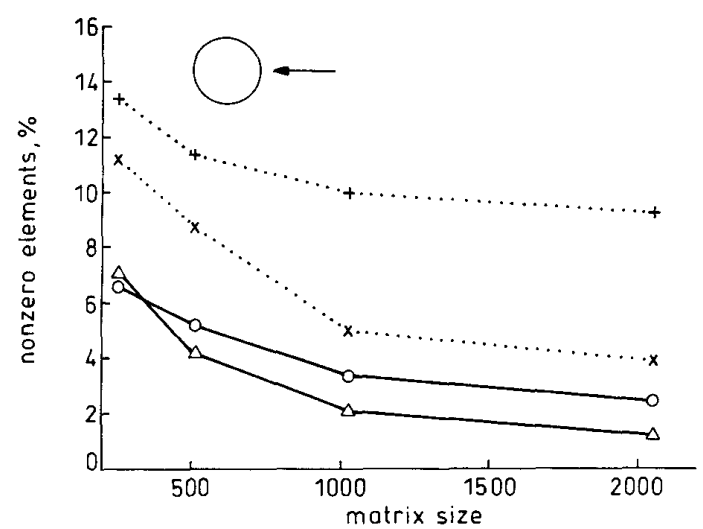

Fig.8 Matrix sparsity as a function of matrix size by WT and WP methods for a cylindrical scatterer

$+\cdots .+.+$ WT by Daubechies 16
$\times \cdots . . \times$ WP by Daubechies 16

- $O$ WT by filter $48 \mathrm{f}$

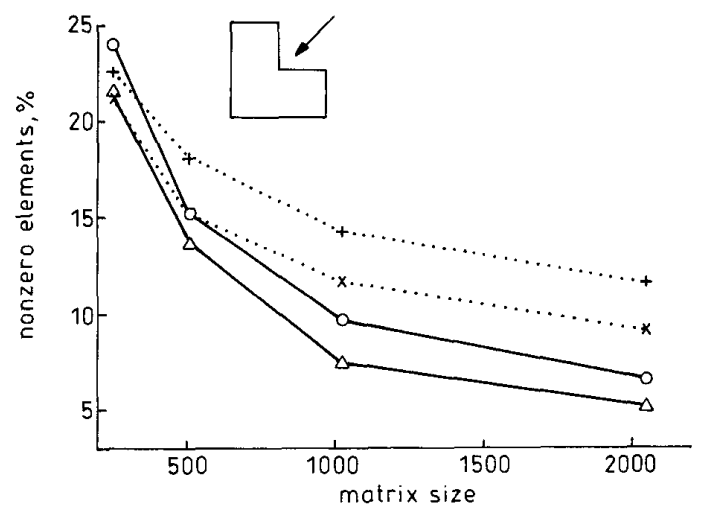

Fig.9 Matrix sparsity as a function of matrix size $N$ by WT and WP methods for an L-shaped scatterer

$+\ldots+$ WT by Daubechies 16

$\times \cdots \times \times$ WP by Daubechies 16

O W W by filter 48

$\triangle$ WP by filter 48

\section{Conclusions}

An effective QMF has been applied to solve the IE solution of 2D EM scattering problems. Compared with the widely used Daubechies wavelets and published data, the QMF with sharper TBW proved to perform well in matrix sparsity. The filter-design approach offers an easy way to select better QMF for EM scattering applications. Using this approach to reduce nonzero elements of the impedance matrix, many types of QMF design can be applied and provide greater degrees of freedom for QMF selection. For electrodynamic applications, a more efficient set of wavelet bases should be constructed to fully capitalise on the potential of wavelets. Some wavelet construction methods in signal/image processing may perform well in computational electromagnetics. Their further development may contribute to the improvement of existing computational EM codes.

\section{Acknowledgments}

This work was sponsored jointly by the Ministry of Education and the National Science Council, ROC, under contract: 89-E-FA06-2-4, and by the Chunghwa Telecom Laboratories, under contact: TL-89-2605. 


\section{References}

1 WAGNER, R.L., and CHEW, W.C.: 'A study of wavelets for the solution of electromagnetic integral equations', IEEE Trans., 1995, AP-43, pp. 802-810

2 GOLIK, W.L.: 'Wavelet packets for fast solution of electromagnetic integral equations', IEEE Trans, 1998, AP-46, (5), pp. 618-624

3 STEINBERG, B.Z., and LEVIATAN, Y.: 'On the use of wavelet expansions in the method of moments', IEEE Trans., 1993, AP-41, pp. $610-619$

4 KIM, $\mathrm{H}$, and $\mathrm{LNG}, \mathrm{H}$. 'On the application of fast wavelet transform to the integral equation solution of electromagnetic scattering problems', Microwave Opt. Technol. Lett., 1993, 6, (3), pp. 168-173

5 Droblems', Microwave Opt. Technol. Lett., 1993, 6, (3), pp. 168-173 adaptive wavelet packet transform', Electron. Lett., 1997, 33, pp. $1127-1128$

6 ZHU, X., LEI, G., and PAN, G.: 'On the application of fast and adaptive periodic Battle-Lemari'e wavelets to modeling of multiple lossy transmission lines', J. Comp. Phys. 1997, 132, pp. 299-311

7 WANG, G.: 'On the utilization of periodic wavelet expansions in moment methods', IEEE Trans., 1995, MTT-43, pp. 2495-2498

8 LEOU, J.-L., HUANG, J.-M., JENG, S.-K., and LI, H.-J.: 'Application of wavelets to scattering problems of inhomogeneous dielectric slabs', IEICE Trans. Commun., 1999, E82-B, (9), pp. 1667-1676

9 PAN, G.: 'Orthogonal wavelets with applications in electromagnetics', IEEE Trans., 1996, MAG-32, pp. 975-982

10 PAN, G., TOUPIKOV, M., DU, J., and GILBERT, B.K.: 'Use of Coifman intervallic wavelets in 2-D and 3-D scattering problems', $I E E$ Proc., 1998, 145, (6), pp. 471 480

11 BEYLKIN, G., COIFMAN, R., and ROKHLIN, V.: 'Fast wavelet transforms and numerical algorithms I', Commun. Pure Appl. Math. 1991, 44, pp. 141-183

12 ALPERT, B., BEYLKIN, G., COIFMAN, R., and ROKHLIN, V.: 'Wavelet-like bases for the fast solution of second-kind integral equations', SIAM J. Sci. Comput., 1993, 14, (1), pp. 159-184

13 GROSSMAN, A., and MORLET, J.: 'Decomposition of Hardy functions into square integrable wavelets of constant shapes', SIAM J. Math. Anal. 1984,15 , pp. 723-736

14 STRANG, G., and NGUYEN, T.: 'Wavelets and filter banks' (Wellesley-Cambridge Press, Wellesley, MA, 1996)
$15 \mathrm{KIM}, \mathrm{H}$., LNG, H., and LEE, C.: 'A fast moment method algorithm using spectral domain wavelet concepts', Radio Sci., 1996, 31, pp. $1253-1261$

16 DAUBECHIES, I.: 'Ten lectures on wavelets' (SIAM, Philadelphia, PA, 1992)

17 SARKAR, TK SU, C ADVE $R$ SALAZAR-PALMA, $M$ GARCIA-CASTILLO, L., and BOIX, R.R.: 'A tutorial on wavelets from an electrical engineering perspective, part 1: discrete wavelet techniques', IEEE Antennas Propag. Mag., 1998, 40, (5), pp. 49-69

18 COHEN, A., DAUBECHIES, I., and FEAUVEAU, J.-C.: 'Biorthogonal bases of compactly supported wavelets', Commun. Pure Appl. Math., 1992, 45, pp. 485-500

19 GOLIK, W.L.: 'Conditioning of biorthogonal wavelet transforms and iterative solvers for electromagnetic integral equations'. Proceedings of 15th annual review of Progress in Applied Computational Electromagnetics, Naval Postgraduate School, Monterey, CA, 1999

20 XIANG, Z., and LU, Y.: 'An effective wavelet matrix transform approach for efficient solutions of electromagnetic integral equations', IEEE Trans., 1997, AP-45, (8), pp. 1205-1213

21 HUANG, J.-M., LEOU, J.-L., JENG, S.-K., and TARNG, J.-H.: 'Application of mix-phase wavelets to sparsify impedance matrix', IEICE Trans. Commun., 1999, E82-B, (9), pp. 1688-1693

22 VAIDYANATHAN, P.P and HUONG, P.-Q. 'Lattice structures for optimal design and robust implementation of two-channel perfectreconstruction QMF banks', IEEE Trans., 1988, ASSP-36, (1), pp. $81-94$

23 WICKERHAUSER, M.V.: 'Adapted wavelet analysis from theory to software' (AK Peters, Ltd, London, 1994)

24 VAIDYANATHAN, P.P.: 'Multirate systems and filter banks' (Prentice-Hall, Englewood Cliffs, NJ, 1993)

25 VAIDYANATHAN, P.P.: 'Passive cascaded lattice structures for low sensitivity FIR filter design, with applications to flter banks', IEEE Trans., 1986, CAS-33, pp. 1045-1064

26 COIFMAN, R.R., and WICKERHAUSER, M.V.: 'Entropy-based algorithms for best basis selection', IEEE Trans., 1992, IT-20, (2), pp. 713-718

27 HARRINGTON, R.F.: 'Time-harmonic electromagnetic fields' (McGraw-Hill, New York, 1961) 